\title{
Utilizing a patient-centric diagnostic and treatment algorithm for pelvic congestion syndrome (PCS) to maximize positive outcomes and patient satisfaction
}

\begin{abstract}
We report our experience utilizing a patient-centric algorithm in diagnosing and treating patients with pelvic congestion syndrome (PCS) in the outpatient surgical setting. 274 cases of PCS were diagnosed and treated at our facility utilizing the described protocol, with preliminary completed patient satisfaction surveys $(\mathrm{n}=26)$. Our observation demonstrates that involving the patient in the diagnosis and treatment of their congestive venous disease has resulted in a high level of consistency in understanding their condition and subsequent satisfaction in this group of patients. Due to the vast presenting symptomatology of PCS, it is clinically important to correlate radiologic findings with presenting symptoms. Through a series of staging procedures, the diagnostic and therapeutic processes were followed until the symptoms causing the pain were resolved. Following treatment for pelvic congestion syndrome utilizing our practice's algorithm, patients reported $83.57 \%$ (76.00 - 91.15\% $\mathrm{CI}=95 \%$ ) resolution of their symptoms.
\end{abstract}

Keywords: pelvic congestion syndrome, chronic pelvic pain, fluoroscopic venography, embolization
Volume 10 Issue I - 2018

\author{
Farber LA,' Kalawur V,',2 Bortecen K,' \\ Rotgans A,' Hollingsworth C,' Tonis A,' \\ Greuner DA' \\ NYC Surgical Associates, USA \\ ${ }^{2}$ Rutgers New Jersey Medical School, USA
}

Correspondence: Leonard Farber MD, NYC Surgical Associates, I 4 E 60th St., Suite 50I, New York, 10022, USA, Tel 888.286.6600, Fax 800.565.9415, Email Ifarber@nycsurgical.net

Received: December 26, 2017| Published: January 17, 2018
Abbreviations: PCS, pelvic congestion syndrome; CTV, computed tomographic venography; MRV, magnetic resonance venography; QOL, quality of life

\section{Introduction}

Pelvic congestion syndrome (PCS) is a common yet often underdiagnosed cause of chronic, i.e., greater than 6months, pelvic pain most often seen in premenopausal, multiparous women. It presents as a constellation of symptoms caused by the development of varicosities in veins typically drained by either the ovarian or internal iliac veins.

Although presentation can be diverse it is commonly described as noncyclical, dull or aching pelvic pain, typically unilateral, and often accompanied by dysmenorrhea and dyspareunia., ${ }^{1,2}$ The underlying pathophysiology of PCS is due to multiple factors but the characteristic pain presentation is thought to be a direct result of varicosities of the pelvis. Valvular insufficiency and venous reflux or obstruction manifesting as ovarian vein or hypogastric vein congestion often result in pelvic varicosities. ${ }^{3}$ External varicosities are not present in all cases of PCS; areas that they are commonly seen include the vulvoperineal, posterior thigh and gluteal regions, and occur in venous regions drained by either the ovarian or internal iliac veins. Patients become symptomatic when the distended and tortuous veins are compressed locally, causing subsequent irritation and inflammation of nearby organs in proximity to the engorged and swollen plexus of pelvic veins.

Pelvic organs including the bladder, vagina, uterus, and rectum, as well as the sacral region are drained by a plexus of pelvic veins and may be affected, prompting a clinical suspicion of PCS. ${ }^{4}$ Some form of bladder instability is not uncommon. Involvement of lumbosacral nerves also may produce a variety of symptoms, including back and hip pain, that can ultimately lead to atypical presentations of PCS. ${ }^{5,6}$ Pressure of these veins on the rectum, or perhaps reflux through collaterals feeding off a refluxing ovarian vein, can result in hemorrhoids. On physical examination, patients often have varicosities on the vulva, on or just beneath the buttocks, and on the upper thighs, particularly near the groin, owing to reflux from the pelvic veins communicating to the legs via the inferior gluteal and internal/ external pudendal veins. ${ }^{4}$ PCS is often a diagnosis of exclusion in the evaluation of chronic pelvic pain and should be considered when workup for more typical causes of pain have been unremarkable, or treatment for other differential diagnoses have proven unsuccessful. PCS is also seen in association with lower extremity varicose veins. As many as $15-20 \%$ of patients with lower limb varicosities have demonstrated pelvic venous reflux on venogram or duplex ultrasound, with a $30 \%$ incidence among those patients with varicosities that have recurred after prior treatment. ${ }^{7}$

Once physical exam findings and traditional imaging modalities exclude the most common causes of intrinsic pelvic pathology and PCS is suspected, a duplex sonography may provide a noninvasive yet diagnostically accurate imaging study in its workup. CT venography (CTV) and magnetic resonance venography (MRV) are other available diagnostic tools, however, they are more expensive, invasive, and in our experience, have failed to be established as being reliable and consistent diagnostic modalities for PCS. This is predicated on the fact that the timing of the imaging in relation to the passage of contrast media through the diseased vessel is inconsistent, and in many instances collateral circulation, especially hypogastric branches that contribute significantly to the etiology of PCS, are not consistently seen. Furthermore, there is a significant user dependence on the technical skillset of the technologist performing the exam as well as that of the diagnostic radiologist. CT venography and magnetic resonance venography also have other limitations that lower their diagnostic yield. ${ }^{3,8,9}$ For instance, both imaging modalities are performed with the patient in a supine position, which can decompress ordinarily dilated veins. In addition, CT venography does not provide hemodynamic physiologic information, for which a localized contrast bolus is needed. Finally, CT venography is also associated with a filling defect in the femoral-to-iliac vein that further limits visualization of pelvic veins. ${ }^{9}$ Pelvic sonography, on the other hand, can detect both dilated pelvic veins and slow or reversed retrograde blood flow. 
This modality is inexpensive, and can be performed with the patient upright, as the examiner looks for both dilated pelvic veins and flow reversal during Valsalva maneuvers. ${ }^{3}$ The operator-dependent nature inherent to sonography mandates valuable sonogram technicians with high levels of training and experience in finding and reporting pelvic reflux and varicosities. As with the CTV and MRV, the sonogram is also capable of ruling out other pelvic etiologies. Findings suggestive of PCS on ultrasound include ovarian veins $>6 \mathrm{~mm}$ in diameter, the presence of dilated $(>5 \mathrm{~mm})$ arcuate veins crossing the uterine myometrium, and slow $(<3 \mathrm{~mm} / \mathrm{sec})$ or reversed retrograde blood flow. ${ }^{3,9-11}$ Polycystic changes in the ovaries have also been seen in about $50 \%$ of cases. ${ }^{11,12}$

With regard to treatment techniques, transcatheter embolization is currently regarded as the least invasive and most efficacious management option for PCS, with complete or partial symptom relief in $68.2-100 \%$ of patients. ${ }^{9}, 13$ A number of different approaches have been reported to affect closure of the refluxing veins, from simple coil embolization, to glue embolization, to combinations of sclerotherapy and coils. In studies using visual analog scale (VAS) pain scores to measure the extent of symptom relief, vast improvement was shown consistently, with mean scores of 7.3-7.6 decreasing to scores of $0.5-3.2$ postoperatively. Depending on the initial severity, the symptoms can take as long as 9-13 months after therapy to resolve. ${ }^{13}$ Complications from the procedure are rare, being reported in $3.4-4.4 \%$ of patients, and consist of coil migration, vein perforation, local phlebitis, deep venous thrombosis, and contrast reactions. ${ }^{13}$ Patients may suffer from a brief period of flu-like symptoms within 72 hours of each embolization, but this consistently passes within 48 hours without need for intervention. Studies have also shown embolization to have no effect on the menstrual cycle or fertility, ${ }^{7}$ which is of clinical significance as PCS is predominantly a disease of premenopausal women

With respect to the management of PCS secondary to stenotic vein lesions, endovenous stent placement is also safe and effective. In a review of multiple studies encompassing 1500 patients being treated for chronic iliac vein stenosis, stenting had a $90-100 \%$ patency rate for non-thrombotic disease and $74-89 \%$ patency rate for thrombotic disease at 3 to 5 years. Symptom relief was achieved in $86-94 \%$ of patients for pain, $66-89 \%$ for swelling, and $58-89 \%$ for healing of ulcers in the leg. ${ }^{14}$ Although these studies focused on the outcomes of patients suffering from venous insufficiency in the legs, it is not unreasonable to assume that treatment of the stenosis would similarly benefit patients with PCS, given that both ailments are due to venous obstruction causing congestion in communicating upstream veins. Among the 1500 patients reviewed, no deaths or pulmonary emboli were reported, and access site complications and significant bleeding, despite the larger sheaths required for the stents, occurred in only $0.03-1 \%{ }^{14}$

\section{Materials and methods}

Patients with chronic pelvic pain, determined by careful history, and physical examination consistent with PCS are consented for treatment and participation in our IRB approved research study with data capture. Patients who qualify undergo initial screening with duplex pelvic sonography. If scanning is positive, they undergo a diagnostic fluoroscopic venogram in the operating room suite. During the initial diagnostic venogram the patient is kept awake and coherent during the examination, a technique often utilized in interventional pain management techniques to ensure that certain pain is reproducible and identifiable as the source. Clinician-patient communication is constant for the duration of the procedure. The initial diagnostic venogram is performed from the right common femoral vein approach as this facilitates the easiest route to cannulation and imaging of the left gonadal vein. This approach is comfortable for the patient, allowing them to visualize their anatomy and communicate during the procedure. Assessment is initiated at the left ovarian vein, the most common site of pelvic reflux. If reflux is present, an attempt is made to reproduce the patient's symptoms using high pressure contrast flushes in a 5 French sheath. If venous distension created by the flush reproduces symptoms, and the anatomic distribution of the vessels distended corresponds to those symptoms, the vessel is then embolized. Our preference in the ovarian vein is to use a combination of gelfoam or polidocanol for the terminal branches below the pelvic brim and an oversized (at least $150 \%$ of the diameter of the ovarian vein) coil in the ovarian vein, preferably near the ostia of any visualized ovarian collaterals, and no less than $4 \mathrm{~mm}$ from its confluence with the renal vein.

During the initial diagnostic venogram an attempt is made to replicate the presenting pain experienced by the patient. Once the patient's pain is replicated, and anatomic location on venogram is correlated with their symptoms, findings are then discussed with the patient. Treatment option of embolization and the rationale for this approach are reviewed while simultaneous demonstration of the venographic results are shown to the patient. Embolization involves utilization of a combination of slurry and an embolic coil made from a bare platinum primary wound coil surrounding a strand of inert hydrogel. Based on our successful clinical experience in gaining fully occlusive embolic results with one coil and less than $10 \mathrm{cc}$ of slurry when both are positioned and delivered appropriately, this has become our standard initial approach. The consistent visual radiographic confirmation of pelvic congestion syndrome intraoperatively in our practice has led to full incorporation of the patient in the process of treating the disease state. This has led to near perfect patient satisfaction scores (article in preparation).

If complete resolution of presenting symptoms is not achieved with our initial diagnostic venogram and embolization procedure, the patient is subsequently taken again to the OR for a right internal jugular approach venogram of the right gonadal vein and hypogastric venous complex, which are evaluated for persistent collaterals. This procedure is typically performed with IV sedation for patient comfort. If reflux is present our same embolic algorithm previously described is employed. If PCS symptoms are not resolved on first embolization, our experience has demonstrated that there may be some level of outflow obstruction contributing to the exasperation of the congestive disease state, either from the renal or iliac systems. If any venographic evidence of stenosis is seen in the left renal or common iliac system, intravascular ultrasound is employed to investigate the level of severity and its impact on post-compressive congestion. Although standard venography of the left renal vein and both left and right internal, external, and common iliac veins are performed, we have found superior diagnostic modality accuracy with IVUS in diagnosing outflow obstructions. If outflow obstruction greater than $50 \%$ is identified the patient is given the option of observation, plain old balloon angioplasty (POBA), or bare metal stent (BMS) therapy. Bare metal stents are not offered to patients contemplating future pregnancy as it has been documented to cause re-occlusive damage in previously placed ileal-caval stents. ${ }^{15}$ For patients greater than 30 years of age with left renal vein stenosis and congestion, we are more apt to simply treat congestion if there is not an additional threat to the kidney, or at least offer the patient a choice given higher risk for migration of left renal stents. For infra-inguinal occlusive lesions, a popliteal vein approach is preferred. This approach has proven largely 
successful in treating infra-inguinal occlusions while expediting a speedy recovery, with excellent access from the popliteal to the IVC. Institutions only treating pelvic veins that are dilated greater than 6 $\mathrm{mm}$ on $\mathrm{CT} / \mathrm{MR}$ venography exclude many patients who could benefit from curative embolic therapy.

Patients are subsequently closely monitored in the postoperative setting. Pain in the immediate postoperative period is controlled with regional nerve block. There is communication with patients the day following their procedure via telephone calls for an update on their clinical status. Any patient with required care is seen in the office. A repeat pelvic sonogram is performed approximately 1 week following the procedure to determine if there is any residual reflux. If noted, a second venogram is performed with subsequent embolization of pelvic varices fed by the refluxing internal iliac veins, and potentially the right ovarian vein if the patient's symptoms are significantly greater on the right. Our preference is to treat the internal iliac branches with either sclerotherapy or gelfoam alone, given the higher risk of coil migration noted in this region. If the right ovarian vein is to be targeted, we consider a right internal jugular access to allow for easier cannulation. During this second procedure, an IVUS catheter is employed to look for stenotic lesions. Pelvic reflux can frequently be secondary to anatomic anomalies resulting in downstream obstruction, such as compression of the left renal vein by the superior mesenteric artery, Nutcracker syndrome, ${ }^{3,7,16}$ or compression of a left common iliac vein by a right common iliac artery, May-Thurner syndrome. ${ }^{1,3,7}$ A May-Thurner's phenomenon can be present in as many as two-thirds of the general population. ${ }^{17}$ If any such lesions are identified, a full discussion with the patient regarding the anatomy affected by the stenosis, and the benefits and drawbacks of angioplasty alone versus stenting ensues.

If the patient consents to proceed, there is a third venogram to treat the stenotic segment(s). For milder lesions $(\leq 50 \%$ reduction in cross-sectional area), or for focal, short lesions caused by a localized vascular band or web, we attempt only an angioplasty. For a longer and more severe stenotic segment $(>50 \%)$, our preference is to perform an angioplasty and stent placement. With regard to utilization of our coils, our preference is to oversize the stents (roughly $4 \mathrm{~mm}$ greater than the average diameter of the non-stenotic venous segment). The goal is to avoid a stenting procedure for as long as possible in women who intend to get pregnant and in patients with clotting disorders or prior DVTs, given the higher incidence of post-stent complications in these patient populations. At the completion of the process and follow up, patients are versed completely on the clinical etiology of their PCS, the approach utilized to treat their condition, and what they can expect as a result of therapy. Having our patients educated in this way has provided our patients with the knowledge base for assessment to determine if additional therapeutic treatment is required. At the conclusion of treatment, patients were given satisfaction surveys to complete.

\section{Results and discussion}

274 cases of pelvic congestion syndrome were diagnosed and treated at our facility utilizing the described protocol, with preliminary completed patient satisfaction surveys $(n=26)$. As consistent with documented historical data, patients presented with a variety of symptoms. The most commonly reported symptoms were back or hip pain $(34.6 \%)$, pelvic pain $(30.8 \%)$, abdominal bloating and heaviness $(23.1 \%)$, and leg pain $(23.1 \%)$. Other symptoms our patients experienced included urinary pain or other urinary symptoms, rectal pain or hemorrhoids, severe menstrual pain, and abdominal pain (Table 1).
After undergoing pelvic congestion treatment with the described algorithm, patients classified their satisfaction with the procedure and treatment as $9.2(8.62-9.78 ; \mathrm{CI}=95 \%)$ out of 10 . Following treatment for pelvic congestion syndrome utilizing our practice's algorithm, patients reported $83.57 \%(76.00-91.15 \% \mathrm{CI}=95 \%)$ resolution of their symptoms (Table 2).

Table I Symptoms of PCS and their prevalence

\begin{tabular}{lll}
\hline Symptom & $\begin{array}{l}\text { Number of } \\
\text { patients }\end{array}$ & $\begin{array}{l}\text { Percent of } \\
\text { patients }\end{array}$ \\
\hline Back or hip pain & 9 & $34.60 \%$ \\
Pelvic pain & 8 & $30.80 \%$ \\
Abdominal bloating and heaviness & 6 & $23.10 \%$ \\
Urinary pain or urinary symptoms & & $23.10 \%$ \\
Leg pain & 6 & $15.40 \%$ \\
Abdominal pain & 4 & $11.50 \%$ \\
Urinary pain or urinary symptoms & 3 & $7.70 \%$ \\
Rectal pain or hemorrhoids & 2 & $3.80 \%$ \\
Severe menstrual pain & 1 & \\
Total & 26 & \\
\hline
\end{tabular}

Table 2 Patient outcomes following treatment for PCS

\begin{tabular}{ll}
\hline Satisfaction Score $(\mathrm{I}-10)$ & $9.2(8.62-9.78 ; \mathrm{Cl}=95 \%)$ \\
\hline Symptom Relief $(0-100 \%)$ & $83.57 \%(76.00-9 \mathrm{I} .15 \% \mathrm{Cl}=95 \%)$
\end{tabular}

The atypical presentation of PCS leads to its underdiagnosis, while being the predominant issue impacting the patient's negative quality of life. These atypical presentations are especially underdiagnosed, which was noted by the extensive workups experienced by 3 patients in our series. ${ }^{5}$ The complexity of the abdominal/pelvic venous vasculature in the premenopausal patient can distort accuracy and effect a patient's ability to properly articulate symptoms. It is therefore important to maintain a low threshold for suspicion of PCS in any patient with symptoms that can be attributed to an organ in the pelvis. With modern advancements made in diagnostic imaging, many clinicians feel MRV to be definitive in diagnosing pelvic congestion, but our experience has proven otherwise. Cross sectional imaging relies heavily on timing, patient immobility and technical skill level of the performing technologist and even then can still provide false positive results. ${ }^{7,9}, 12$ Our experience has shown us that fluoroscopic venography is the gold standard diagnostic imaging modality providing anatomical correlation as well as physiological confirmation of our suspicions. In all of our patients, pelvic venograms successfully identified the condition of PCS

Given the effectiveness and safety of endovenous stent placement procedures, our practice utilized aggressive management of patients whose symptoms and venogram findings suggest the presence of PCS. Using an algorithm of staging procedures, the diagnostic and therapeutic processes were followed until the symptoms causing the pain were resolved. Patients were involved in the process which proved to result in a more positive outcome with regard to patient satisfaction.

The high QOL scores of our documented treated patients are an objective metric that speaks to the efficacy, safety, and accuracy of our treatment methodology. Although 274 patients were treated in this method at the time of the paper, only 26 had completed the satisfaction surveys. Updated surveys are currently being collected and the data to be subsequently reported. However, preliminary reports are encouraging and warrant further follow-up and analysis. Our diagnostic and treatment approach revolves around the patient perspective, taking into consideration logistical, emotional, and 
clinical variables, and the full patient involvement in every step of the process from diagnosis to treatment, recovery, and follow-up. Involving patients in this process has allowed for a more positive outcome with regard to patient satisfaction.

\section{Conclusion}

The diagnosis of pelvic congestion syndrome continues to remain a challenge to physicians in multiple specialties due to the vast presenting symptoms. Often other pathological conditions are first ruled out and PCS becomes a diagnosis of exclusion, although fluoroscopic venography as a primary diagnostic modality may ultimately serve to establish diagnosis. It is therefore important to maintain a high level of suspicion in parous women whether presenting symptoms are typical or atypical in nature. Incorporating patient response during both the diagnostic and therapeutic processes leads to improvement in pain symptomatology and overall patient satisfaction with their procedures and outcome.

\section{Acknowledgments}

The authors would like to thank the receptionist staff at NYC Surgical Associates for their help in distributing and collecting patient satisfaction surveys.

\section{Conflicts of interest}

The authors declare no conflict of interest.

\section{References}

1. Liddle AD, Davies AH. Pelvic congestion syndrome:chronic pelvic pain caused by ovarian and internal iliac varices. Phlebology. 2007;22(3):100-104.

2. Black CM, Thorpe K, Venrbux A, et al. Research reporting standards for endovascular treatment of pelvic venous insufficiency. $J$ Vasc Interv Radiol. 2010;21(6):796-803.

3. Durham JD, Machan L. Pelvic congestion syndrome. Semin Intervent Radiol. 2013;30(4):372-380.

4. Stones RW. Pelvic vascular congestion:half a century later. Clin Obstet Gynecol. 2003;46(4):831-836.
5. Greuner D, Amorosso A, Rotgans A, et al. Atypical presentations and treatment variations of pelvic congestion syndrome:a four patient case series. F1000Research. 2006;5:2906.

6. Shelkey J, Huang C, Karpa K, et al. Case report:pelvic congestion syndrome as an unusual etiology for chronic hip pain in 2 active, middle-age women. Sports Health. 2014;6(2):145-148.

7. Lopez AJ. Female pelvic vein embolization:indications, techniques, and outcomes. Cardiovasc Intervent Radiol. 2015;38(4):806-820.

8. Koo S, Fan CM. Pelvic congestion syndrome and pelvic varicosities. Tech Vasc Interventional Rad. 2014;17(2):90-95.

9. Sato K, Orihashi K, Takahashi S, et al. Three-dimensional CT Venography:a diagnostic modality for the preoperative assessment of patients with varicose veins. Ann Vasc Dis. 2011;4(3):229-234.

10. Park SJ, Lim JW, Ko YT, et al. Diagnosis of pelvic congestion syndrome using transabdominal and transvaginal sonography. AJR Am J Roentgenol. 2004;182(3):683-688.

11. Ignacio EA, Dua R, Sarin S, et al. Pelvic congestion syndrome:diagnosis and treatment. Semin Intervent Radiol. 2008;25(4):361-368.

12. Bittles MA, Hoffer EK. Gonadal vein embolization:treatment of varicocele and pelvic congestion syndrome. Semin Intervent Radiol. 2008;25(3):261-270.

13. Marsh P, Holdstock J, Harrison C, et al. Pelvic vein reflux in female patients with varicose veins:comparison of incidence between a specialis private vein clinic and the vascular department of a National Health Service District General Hospital. Phlebology. 2009;24(3):108-113.

14. Raju S, Darcey R, Neglén P. Unexpected major role for venous stenting in deep reflux disease. J Vasc Surg. 2010;51(2):401-408.

15. Hartung $\mathrm{O}$, Barthelemy $\mathrm{P}$, Arnoux $\mathrm{D}$, et al. Management of pregnancy in women with previous ilio-caval stenting. $J$ Vasc Surg. 2009;50(2):355-359.

16. Greiner M, Dadon M, Lemasle P, et al. How does the patho-physiology influence the treatment of pelvic congestion syndrome and is the result long-lasting? Phlebology. 2012;27(Suppl 1):58-64.

17. Raju S. Best management options for chronic iliac vein stenosis and occlusion. J Vasc Surg. 2013;57(4):1163-1169. 